\title{
A decoding-based fusion rule for cooperative spectrum sensing with nonorthogonal transmission of local decisions
}

\author{
Simin Bokharaiee ${ }^{1}, \mathrm{Ha} \mathrm{H}_{\mathrm{Nguyen}}{ }^{2^{*}}$ and Ed Shwedyk ${ }^{1}$
}

\begin{abstract}
Cooperative spectrum sensing in cognitive radio (CR) networks is studied in which each CR performs energy detection to obtain a binary decision on the absence/presence of the primary user. The problem of interest is how to efficiently report and combine the local decisions to/at the fusion center under fading channels. In order to reduce the required transmission bandwidth in the reporting phase, the paper examines nonorthogonal transmission of local decisions by means of on-off keying. Proposed and analyzed is a novel decoding-based fusion rule that essentially performs in three steps: (1) estimating minimum mean-square error of the transmitted information from cognitive radios, (2) making hard decisions of the transmitted bits based on the estimated information, and (3) combining the hard decisions in a linear manner. Simulation results support the theoretical analysis and show that the added complexity of the decoding-based fusion rule leads to a considerable performance gain over the simpler energy-based fusion rule when the reporting links are reasonably strong.
\end{abstract}

Keywords: Cognitive radio; Spectrum sensing; Likelihood ratio test; Energy detection; On-off keying; Noncoherent transmission; Signature sequences

\section{Introduction}

Cognitive radio $(\mathrm{CR})$ is an attractive technology to deal with the spectrum scarcity issue as the number of wireless applications and systems grows quickly. The main principle behind cognitive radio is to provide wireless access to potential users by opportunistically detecting the unused licensed bands, originally allocated to some primary users. The key for enabling such an opportunistic access lies in a reliable spectrum sensing technique. The technique of distributed spectrum sensing, in which the observations of CR nodes are collected and transmitted to a fusion center (FC) for a final sensing decision, has received a great interest in recent years. The fusion center aggregates the information pieces transmitted from the CRs and combines them according to some fusion rule in order to make a final decision about the absence (denoted by $\mathcal{H}_{0}$ ) or presence (denoted by $\mathcal{H}_{1}$ ) of the primary user in the band of the interest.

\footnotetext{
*Correspondence: ha.nguyen@usask.ca

${ }^{2}$ Department of Electrical and Computer Engineering, University of Saskatchewan, 57 Campus Drive, Saskatoon, Saskatchewan S7N 5A9, Canada Full list of author information is available at the end of the article
}

Since the transmission of the local sensing data to the FC can be costly in terms of bandwidth requirement, particularly for large-scale distributed CR networks, some form of local data compression is preferred in which each CR sends to the FC only one or a few bits of data, representing its local sensing result. To further reduce bandwidth consumption while maintaining simple fusion processing, nonorthogonal transmission of local decisions can be employed by means of on-off keying (OOK) [1]. In such a transmission technique, the CRs are allocated with nonorthogonal (correlated) signature vectors (SVs). If the length of the signature vectors is substantially less than the number of CRs, the bandwidth efficiency can be significantly improved $[1,2]$.

Our earlier work in [3] proposes a collaborative weighted energy-based fusion rule with noncoherent transmission of 1-bit decisions and when the sensing results are reported to the FC over orthogonal channels. The main focus of the work in [3] is to optimize the sensing thresholds at the local CRs, the combining gains at the fusion center, and the sensing time to maximize

\section{望 Springer}


the secondary throughput of a CR network. However, for large-scale CR networks, assigning orthogonal channels to all CRs might lead to an unaffordable bandwidth expenditure.

This paper adopts the same nonorthogonal transmission framework of [1] for the reporting phase. Different from [1], our main contribution is to develop a lowcomplexity decoding-based fusion rule as an alternative to the energy-based fusion rule in order to efficiently suppress the noise in the received signal at the fusion center and achieve a better sensing performance when the reporting channels are strong. The performance of the proposed fusion rule is analyzed and compared with the performance of the energy-based fusion rule under different scenarios.

The remaining of the paper is organized as follows. Section 2 introduces the model of cooperative spectrum sensing with nonorthogonal transmission of local decisions. Section 3 summarizes the energy-based fusion rule, whereas the decoding-based fusion rule is developed and analyzed in Section 4. Simulation results are presented and discussed in Section 5. Finally, Section 6 draws conclusions.

\section{System model}

The structure of cooperative spectrum sensing under consideration is illustrated in Figure 1. There is one primary source, $K$ CR nodes, and one fusion center. For simplicity, the primary source is indexed by node 0 , CR nodes are denoted by nodes $i, i=1, \ldots, K$, and the fusion center is identified as node $K+1$.

During the sensing period, each CR collects its observations from the primary user's signal in order to make a local decision on the binary hypothesis $\mathcal{H}_{0}$ or $\mathcal{H}_{1}$. Due to the presence of Rayleigh fading channels between the primary user and CRs, the local observations at CRs can be treated as independent and identically distributed (i.i.d.) random variables. For processing the observations at each CR, an energy detector (which is known as an optimal detector for i.i.d. signals) is implemented. In particular, local binary decisions are obtained by comparing the energy of the collected signals to a sensing threshold.

In the reporting phase, the local decisions are transmitted to the fusion center over Rayleigh fading channels. For such transmission, the same framework presented in [1] is adopted. The local decision at the $k$ th $C R$ is multiplied (i.e., modulated) with a unique signature vector $\boldsymbol{g}_{k}$ whose length is $M<K$. All the $K$ 'modulated' signature vectors are then transmitted simultaneously in $M$ chip intervals to the fusion center. As mentioned before, the main reason for having $M<K$ is to reduce the transmission bandwidth when compared to the case of $M=K$, i.e., orthogonal transmission. The latter has been studied in [3].

The following detailed description of signal processing at CRs in the sensing phase is basically the same as that in [3], and it is given for completeness. The received signal at each CR is first passed to an ideal band-pass (BP) filter in order to limit the average noise power as described in [4]. The low-pass equivalent of the output of the BP filter at $\mathrm{CR}$ node $i, i=1, \ldots, K$, can be represented as

$$
\begin{aligned}
& x_{0, i}\left(t \mid \mathcal{H}_{0}\right)=n_{0, i}(t), \\
& x_{0, i}\left(t \mid \mathcal{H}_{1}\right)=\sqrt{E_{0}} h_{0, i} x(t)+n_{0, i}(t),
\end{aligned}
$$

where $x(t)$ denotes the low-pass equivalent of the transmitted signal from the primary user, $E_{0}$ is the average transmitted symbol energy of the source, and $h_{0, i} \sim$ $\mathcal{C N}\left(0, \sigma_{0, i}^{2}\right)$ denotes the coefficient of the fading channel between node 0 (primary user) and CR node $i$. The notation $\mathcal{C N}(\mathbf{m}, \boldsymbol{\Sigma})$ refers to a complex Gaussian random vector (or variable) with mean vector $\mathbf{m}$ and covariance matrix $\boldsymbol{\Sigma}$. Also, $n_{0, i}(t)$ is the filtered additive white Gaussian noise process.

\section{SYSTEM MODEL}

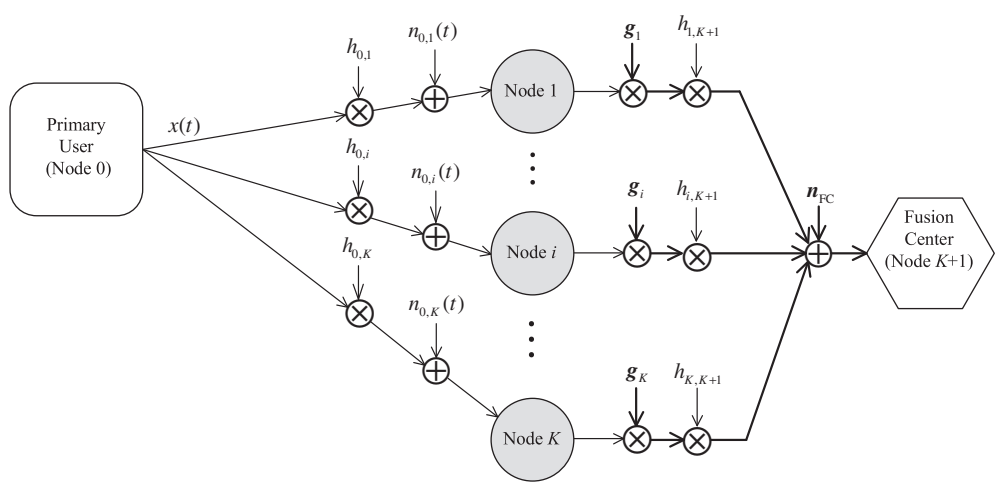

Figure 1 Structure of cooperative spectrum sensing with nonorthogonal transmission in the reporting phase. 
The received signal $x_{0, i}(t)$ at each node is sampled with a rate of $f_{s}=\frac{1}{T_{s}} \mathrm{~Hz}$ over a time duration of $\tau=$ $N T_{\mathrm{s}}$ seconds, where $T_{\mathrm{s}}$ denotes the sampling period and $N$ denotes the number of samples collected for spectrum sensing. Let $x_{0, i}[n]$ be the sample value at the sampling time index $n$. Then, using (1) and (2), it can be expressed as

$$
\begin{aligned}
& x_{0, i}\left[n \mid \mathcal{H}_{0}\right]=n_{0, i}\left(n T_{s}\right) \\
& x_{0, i}\left[n \mid \mathcal{H}_{1}\right]=\sqrt{E_{0}} h_{0, i} x\left(n T_{s}\right)+n_{0, i}\left(n T_{s}\right), \quad 1 \leq n \leq N,
\end{aligned}
$$

where $n_{0, i}\left(n T_{s}\right)$ are the samples of $n_{0, i}(t)$, which can be shown to be i.i.d. complex Gaussian random variables with mean 0 and variance $\sigma_{\mathrm{CR}}^{2}=N_{0} W$ [4-6]. Here, $N_{0}$ is the two-sided power spectral density (PSD) of the white noise before the band-pass filter, and $W$ is the bandwidth of the band-pass filter.

Without loss of generality, the noise variance at each CR node can be normalized to 1 , i.e., $\sigma_{\mathrm{CR}}^{2}=1$. Then, the distribution of the received sample at each CR node is

$$
\begin{aligned}
x_{0, i}\left[n \mid \mathcal{H}_{0}\right] & \sim \mathcal{C N}(0,1) \\
x_{0, i}\left[n \mid \mathcal{H}_{1}\right] & \sim \mathcal{C N}\left(0, \gamma_{i}+1\right),
\end{aligned}
$$

where $\gamma_{i} \triangleq \frac{E_{0} \sigma_{0, i}^{2}}{\sigma_{\mathrm{CR}}^{2}}$ is the received signal-to-noise ratio (SNR) at each node. After collecting $N$ signal samples, each of the CRs obtains its test statistics as follows:

$$
y_{i}=\frac{1}{N} \sum_{n=1}^{N}\left|x_{0, i}[n]\right|^{2}, i=1, \ldots, K
$$

In essence, the above test statistics is a measure of the average energy of the band-limited signal at each CR node over a duration of $\tau$ seconds where $\tau=\frac{N}{f_{s}}$ and $f_{s}$ is the sampling rate. When the number of collected samples, $N$, is large, the central limit theorem can be applied to model $y_{i}$ under both hypotheses with Gaussian distributions [7-10]. That is, $f\left(y_{i} \mid \mathcal{H}_{0}\right) \sim \mathcal{N}\left(1, \frac{1}{N}\right)$, and $f\left(y_{i} \mid \mathcal{H}_{1}\right) \sim$ $\mathcal{N}\left(\gamma_{i}+1, \frac{\left(\gamma_{i}+1\right)^{2}}{N}\right)$. Here, the notation $\mathcal{N}(\mathbf{m}, \boldsymbol{\Sigma})$ means a real Gaussian random vector (or variable) with mean vector $\mathbf{m}$ and covariance matrix $\boldsymbol{\Sigma}$.

The decision device at the $i$ th $C R$ node produces a binary decision ( 0 or 1 , corresponding to hypothesis $\mathcal{H}_{0}$ or $\mathcal{H}_{1}$, respectively) by comparing $y_{i}$ to a decision threshold $\epsilon_{i}$ as follows:

$$
u_{i}=\left\{\begin{array}{l}
0, \text { if } y_{i}<\epsilon_{i} \\
1, \text { if } y_{i} \geq \epsilon_{i}
\end{array}\right.
$$

Let $p_{f_{i}}$ and $p_{d_{i}}$ denote the probability of false alarm and the probability of detection, respectively, at the $i$ th CR node. They can be obtained as [7]

$$
\begin{gathered}
p_{f_{i}}=\operatorname{Pr}\left(y_{i} \geq \epsilon_{i} \mid \mathcal{H}_{0}\right)=Q\left(\left(\epsilon_{i}-1\right) \sqrt{\tau f_{s}}\right), \\
p_{d_{i}}=\operatorname{Pr}\left(y_{i} \geq \epsilon_{i} \mid \mathcal{H}_{1}\right)=Q\left(\left(\frac{\epsilon_{i}}{\gamma_{i}+1}-1\right) \sqrt{\tau f_{s}}\right) .
\end{gathered}
$$

After making a local binary decision, if the CR decides $\mathcal{H}_{1}$ (i.e., $u_{i}=1$ ), the signal vector to be transmitted to the fusion center is obtained as the product of $a_{i}$ and the $M \times 1$ signature vector $\boldsymbol{g}_{i}$. All the signature vectors have unit energy, i.e., $\left\|\boldsymbol{g}_{i}\right\|^{2}=1, i=1, \ldots, K$. On the other hand, if a node decides $\mathcal{H}_{0}\left(u_{i}=0\right)$, it remains silent and does not send a signal to the fusion center. Equivalently, the transmission scheme can also be viewed as a censoring scheme, where only the CRs with nonzero decisions transmit [1]. The transmitted signal from the $i$ th CR can simply be expressed as $v_{i} \boldsymbol{g}_{i}=\left(a_{i} u_{i}\right) \boldsymbol{g}_{i}$, where $v_{i}=a_{i} u_{i}$. It should be noted that the parameter $a_{i}$ sets the average transmitted power of the $i$ th CR. Here, we assume that all CRs are similar, and without loss of generality, they can transmit with an average gain of $a_{i}=1$. For the case of $M=K$, one can choose $\boldsymbol{g}_{i}=\boldsymbol{e}_{i}$, where $\boldsymbol{e}_{i}$ is a column vector of length $K$ with the $i$ th element equal to 1 and all other elements equal to 0 . Obviously, the choice leads to orthogonal transmission of OOK modulated signals, which has been treated in [3]. In contrast, the main focus of this paper is the case when $M<K$ and the SVs cannot be made orthogonal. The key benefit of using shorter SVs is that the transmission in the reporting phase can be conducted with a smaller bandwidth.

After all the $K$ signal vectors are transmitted in $M$ chip intervals to the fusion center over independent Rayleigh fading channels, the $M \times 1$ received signal vector $z=$ $\left[z_{1}, \ldots, z_{M}\right]^{\top}$ at the fusion center is given as

$$
\boldsymbol{z}=\sum_{i=1}^{K} h_{i, K+1} v_{i} \boldsymbol{g}_{i}+\boldsymbol{n}_{\mathrm{FC}},
$$

where $h_{i, K+1} \sim \mathcal{C N}\left(0, \sigma_{i, K+1}^{2}\right)$ represents the channel fading coefficient between the $i$ th $\mathrm{CR}$ and the fusion center, and $\boldsymbol{n}_{\mathrm{FC}}=\left[n_{1, \mathrm{FC}}, \ldots, n_{M, \mathrm{FC}}\right]^{\top} \sim \mathcal{C N}\left(\mathbf{0}, \sigma_{\mathrm{FC}}^{2} \mathbf{I}\right)$ is the $M \times 1$ noise vector at the FC. Let $\boldsymbol{G}=\left[\boldsymbol{g}_{1}, \boldsymbol{g}_{2}, \ldots, \boldsymbol{g}_{K}\right]$ be a $M \times K$ matrix whose columns are the SVs of the CRs, $\boldsymbol{H}$ be a $K \times K$ diagonal matrix whose diagonal entries are $\left\{h_{1, K+1}, h_{2, K+1}, \ldots, h_{K, K+1}\right\}$, and define $\boldsymbol{v}=$ $\left[v_{1}, v_{2}, \ldots, v_{K}\right]^{\top}$. Then, (10) can also be written as

$$
\boldsymbol{z}=\boldsymbol{G H} \boldsymbol{v}+\boldsymbol{n}_{\mathrm{FC}}
$$

The next sections examine two fusion rules, namely, the energy-based fusion rule and the decoding-based fusion rule. In fact, the simple energy-based fusion rule was also discussed in [1]. However, its analysis does not explicitly take into account the signal processing at the CRs 
and cannot be used for parameter optimizations. On the other hand, the decoding-based fusion rule presented in this paper is novel and offers an attractive performancecomplexity tradeoff when compared to the simple energybased fusion rule or the optimum fusion rule in [1]. It should also be pointed out that the optimum fusion rule in [1] not only has the complexity that is exponential in the number of CRs, but is also difficult to analyze for the purpose of parameter optimizations. Similar to [3] and [8], the objective of optimizing parameters for a fusion rule is to maximize the secondary throughput while maintaining the probability of detection equal or above a target value $\bar{P}_{\mathrm{D}}$. For a given sensing time, maximizing the throughput function for a target $\bar{P}_{\mathrm{D}}$ is equivalent to minimizing the probability of false alarm.

\section{Energy-based fusion rule}

Let $\Upsilon=\frac{z^{H} z}{\sigma_{\mathrm{FC}}^{2}}$ denote the normalized output of the energy detector. The energy-based fusion rule is simply

$$
\Upsilon \stackrel{\mathcal{H}_{1}}{\underset{\mathcal{H}_{0}}{\gtrless}} \lambda^{(\mathrm{E})}
$$

where $\lambda^{(\mathrm{E})}$ is the decision threshold at the FC.

Although $\Upsilon$ has a quadratic form of zero-mean Gaussian random variables, we shall approximate it by a Gaussian distribution. The mean and variance of random variable $\Upsilon$ under each hypothesis are obtained as follows. First, it follows from (11) that

$$
\mu_{0} \triangleq \mathcal{E}\left[\Upsilon \mid \mathcal{H}_{0}\right]=\sum_{i=1}^{K} \xi_{i} p_{f_{i}}\left\|\boldsymbol{g}_{i}\right\|_{2}^{2}+M=\sum_{i=1}^{K} \xi_{i} p_{f_{i}}+M
$$

$$
\mu_{1} \triangleq \mathcal{E}\left[\Upsilon \mid \mathcal{H}_{1}\right]=\sum_{i=1}^{K} \xi_{i} p_{d_{i}}\left\|\boldsymbol{g}_{i}\right\|_{2}^{2}+M=\sum_{i=1}^{K} \xi_{i} p_{d_{i}}+M,
$$

where $\xi_{i} \triangleq \frac{\sigma_{i, K+1}^{2}}{\sigma_{\mathrm{FC}}^{2}}$.

Next, the variance is calculated as $\operatorname{var}[\Upsilon]=$ $\mathcal{E}\left[\Upsilon^{2}\right]-(\mathcal{E}[\Upsilon])^{2}$. Using (11), one has

$$
\begin{aligned}
\mathcal{E}\left[\Upsilon^{2}\right]=\mathcal{E} & {\left[\sum_{i, j=1, i \neq j}^{K} u_{i}^{2} u_{j}^{2} \xi_{i} \xi_{j}\left(\boldsymbol{g}_{i}^{H} \boldsymbol{g}_{j}\right)^{2}+2 \sum_{i=1}^{K} u_{i}^{4} \xi_{i}^{2}\right.} \\
& +\sum_{i, j=1, i \neq j}^{K} u_{i}^{2} u_{j}^{2} \xi_{i} \xi_{j}+M+M^{2} \\
& \left.+(2+2 M) \sum_{i=1}^{K} u_{i}^{2} \xi_{i}\right]
\end{aligned}
$$

Then, the variance conditioned on each hypothesis is

$$
\begin{aligned}
v_{0}^{2} \triangleq \operatorname{var}\left[\Upsilon \mid \mathcal{H}_{0}\right]= & \sum_{i, j=1, i \neq j}^{K} p_{f_{i}} p_{f_{j}} \xi_{i} \xi_{j}\left(\boldsymbol{g}_{i}^{H} \boldsymbol{g}_{j}\right)^{2}+2 \sum_{i=1}^{K} p_{f_{i}} \xi_{i}^{2} \\
& +\sum_{i, j=1, i \neq j}^{K} p_{f_{i}} p_{f_{j}} \xi_{i} \xi_{j}+M+M^{2} \\
& +(2+2 M)\left(\sum_{i=1}^{K} p_{f_{i}} \xi_{i}\right)-\mu_{0}^{2} \\
v_{1}^{2} \triangleq \operatorname{var}\left[\Upsilon \mid \mathcal{H}_{1}\right]= & \sum_{i, j=1, i \neq j}^{K} p_{d_{i} p_{d j} \xi_{i} \xi_{j}\left(\boldsymbol{g}_{i}^{H} \boldsymbol{g}_{j}\right)^{2}+2 \sum_{i=1}^{K} p_{d_{i}} \xi_{i}^{2}} \\
& +\sum_{i, j=1, i \neq j}^{K} p_{d i} p_{d j} \xi_{i} \xi_{j}+M+M^{2} \\
& +(2+2 M)\left(\sum_{i=1}^{K} p_{d i} \xi_{i}\right)-\mu_{1}^{2} .
\end{aligned}
$$

It is noted that only the conditional variances depend on the set of SVs. Furthermore, for the special case of orthogonal transmission, one can easily verify that the means and variances reduce to the expressions given in [3].

The probability of false alarm and probability of detection are then obtained as

$$
P_{\mathrm{F}}^{(\mathrm{E})}\left(\boldsymbol{p}_{\boldsymbol{f}}, \lambda^{(\mathrm{E})}\right)=\operatorname{Pr}\left(\Upsilon>\lambda^{(\mathrm{E})} \mid \mathcal{H}_{0}\right)=Q\left(\frac{\lambda^{(\mathrm{E})}-\mu_{0}}{\nu_{0}}\right)
$$

and

$$
P_{\mathrm{D}}^{(\mathrm{E})}\left(\boldsymbol{p}_{\boldsymbol{d}}, \lambda^{(\mathrm{E})}\right)=\operatorname{Pr}\left(\Upsilon>\lambda^{(\mathrm{E})} \mid \mathcal{H}_{1}\right)=Q\left(\frac{\lambda^{(\mathrm{E})}-\mu_{1}}{v_{1}}\right) .
$$

From (18) and (19), for a target $\bar{P}_{\mathrm{D}}$, we obtain

$$
P_{\mathrm{F}}^{(\mathrm{E})}\left(\boldsymbol{p}_{\boldsymbol{f}}, \bar{P}_{\mathrm{D}}\right)=Q\left(\frac{Q^{-1}\left(\bar{P}_{\mathrm{D}}\right) v_{1}+\mu_{1}-\mu_{0}}{v_{0}}\right) .
$$

For a given set of SVs, it is of interest to find the optimal sensing thresholds at the cognitive radios in order to minimize $P_{\mathrm{F}}^{(\mathrm{E})}\left(\boldsymbol{p}_{\boldsymbol{f}}, \bar{P}_{\mathrm{D}}\right)$ in $(20)$. The solution for the case of low sensing SNR, i.e., $\gamma_{i} \ll 1$ can be derived in a similar way as in [3]. The result is

$$
\epsilon_{i}^{*}=\left(1+\frac{1}{\gamma_{i}}\right) \log \left(\gamma_{i}+1\right) .
$$

Furthermore, one can also try to find the set of signature vectors to further minimize $P_{\mathrm{F}}^{(\mathrm{E})}\left(\boldsymbol{p}_{\boldsymbol{f}}, \bar{P}_{\mathrm{D}}\right)$. Unfortunately, 
such an optimization problem in its general form appears to be very complex, and finding a closed-form solution for optimal $\boldsymbol{G}$ seems intractable. Nevertheless, a good set of signature vectors can be found by generating a large set of random signature vectors and picking the one that maximizes the expression in (20).

For the simple case when the sensing channels as well as the reporting channels have the same average SNRs, i.e., $\gamma_{i}=\gamma$ and $\xi_{i}=\xi$, it can be shown that the so-called Welch-bound equality (WBE) sequences [11] yield the optimal signature vectors. The proof is as follows: Observe from (16) and (17) that both $v_{0}$ and $v_{1}$ are functions of the total squared correlation (TSC), namely $\sum_{i, j=1, i \neq j}^{K}\left(\boldsymbol{g}_{i}^{H} \boldsymbol{g}_{j}\right)^{2}$. Then, in order to maximize $\frac{Q^{-1}\left(\bar{P}_{\mathrm{D}}\right) \nu_{1}+\mu_{1}-\mu_{0}}{\nu_{0}}$, it is clear that $\sum_{i, j=1, i \neq j}^{K}\left(\boldsymbol{g}_{i}^{H} \boldsymbol{g}_{j}\right)^{2}$ has to be minimized. It is well known that the WBE sequences minimize the TSC [2].

\section{Decoding-based fusion rule}

Though being very simple, the energy-based fusion rule might not work well in certain channel conditions. This is because noise is also included in the energy calculation. To see how one can improve the performance of the energy-based fusion rule, define $\boldsymbol{D}=$ $\operatorname{diag}\left(a_{1}^{2}|h|_{1, K+1}^{2}, \ldots, a_{K}^{2}|h|_{K, K+1}^{2}\right)$. Then, the energy computed at the FC can be written as

$$
\boldsymbol{z}^{H} \boldsymbol{z}=\boldsymbol{u}^{T} \boldsymbol{D} \boldsymbol{G}^{T} \boldsymbol{G} \boldsymbol{D} \boldsymbol{u}+\boldsymbol{n}_{\mathrm{FC}}^{H} \boldsymbol{n}_{\mathrm{FC}}+2 \boldsymbol{u}^{T} \boldsymbol{D} \boldsymbol{G}^{T} \boldsymbol{n}_{\mathrm{FC}} .
$$

As can be seen, $z^{H} z$ is actually a weighted sum of the local decisions and noise terms. This suggests that one might decode the received signal first and then combine the hard decisions in the hope of achieving a better performance due to better noise reduction under certain channel conditions.

To develop a decoding-based fusion rule, it is proposed to perform the minimum mean-square error (MMSE) estimation of the transmitted vector $\boldsymbol{v}_{\boldsymbol{h}} \triangleq \boldsymbol{H} \boldsymbol{v}$ first and followed by a detection of the transmitted bits from the estimated vector $\hat{\boldsymbol{v}}_{\boldsymbol{h}}$. The MMSE estimation yields [12]

$$
\hat{v}_{h}=C z
$$

where $\boldsymbol{C} \triangleq \overline{\boldsymbol{D}} \boldsymbol{G}^{T}\left[\boldsymbol{G} \overline{\boldsymbol{D}} \boldsymbol{G}^{T}+\sigma_{\mathrm{FC}}^{2} \boldsymbol{I}\right]^{-1}, \overline{\boldsymbol{D}}=\operatorname{diag}\left(\bar{d}_{1}, \ldots, \bar{d}_{K}\right)$ with $\bar{d}_{i}=a_{i}^{2} \sigma_{i, K+1}^{2} \kappa_{i}$ and $\kappa_{i} \triangleq\left[p_{f_{i}} \operatorname{Pr}\left(\mathcal{H}_{0}\right)+p_{d_{i}} \operatorname{Pr}\left(\mathcal{H}_{1}\right)\right]$. It is not hard to show the following relationship between $\hat{\boldsymbol{v}}_{\boldsymbol{h}}$ and $\boldsymbol{v}_{\boldsymbol{h}}$ :

$$
\hat{\boldsymbol{v}}_{\boldsymbol{h}}=\boldsymbol{v}_{\boldsymbol{h}}+\chi
$$

where $\hat{\boldsymbol{v}}_{\boldsymbol{h}}=\left[\hat{\boldsymbol{v}}_{\boldsymbol{h}, 1}, \ldots, \hat{\boldsymbol{v}}_{\boldsymbol{h}, K}\right]^{T}$ and $\boldsymbol{\chi}=\left[\chi_{1}, \ldots, \chi_{K}\right]$ is a zero-mean Gaussian vector. More importantly, the normalized MMSE for each decoded bit can be shown to be

$$
\frac{\mathcal{E}\left[\left(\hat{v}_{\boldsymbol{h}, i}-v_{\boldsymbol{h}, i}\right)^{2}\right]}{\bar{d}_{i}}=1-\boldsymbol{g}_{i}^{T}\left(\boldsymbol{G} \overline{\boldsymbol{D}} \boldsymbol{G}^{T}+\sigma_{\mathrm{FC}}^{2} \boldsymbol{I}\right)^{-1} \boldsymbol{g}_{i} \bar{d}_{i}
$$

In general, the MMSE is a useful performance measure for parameter estimation. In many research papers concerning the detection performance over overloaded code-division multiple access systems, signature vectors are obtained by minimizing MMSE-related metrics, and WBE sequences (or weighted WBE sequences) turn out to be the optimum signature vectors $[2,12]$. However, in the spectrum sensing problem, the main performance measures are mainly related to the probability of false alarm and probability of detection, and the WBE sequences are not necessarily the ones that optimize the performance.

The influence of the choice of the SVs on the performance of the proposed decoding-based fusion rule can be analyzed as follows. First, the likelihood ratio test (LRT) for decoding the bits sent by the $i$ th $\mathrm{CR}$ is

$$
\begin{aligned}
\frac{f\left(\hat{v}_{\boldsymbol{h}, i} \mid u_{i}=1\right)}{f\left(\hat{v}_{\boldsymbol{h}, i} \mid u_{i}=0\right)} \stackrel{\hat{u}_{i}=1}{\gtrless} \frac{\operatorname{Pr}\left(u_{i}=0\right)}{\operatorname{Pr}\left(u_{i}=1\right)} \\
=\frac{1-p_{f_{i}} \operatorname{Pr}\left(\mathcal{H}_{0}\right)-p_{d_{i}} \operatorname{Pr}\left(\mathcal{H}_{1}\right)}{p_{f_{i}} \operatorname{Pr}\left(\mathcal{H}_{0}\right)+p_{d_{i}} \operatorname{Pr}\left(\mathcal{H}_{1}\right)},
\end{aligned}
$$

where the above threshold (i.e., the term on the right hand side of (26)) is obtained by having $\operatorname{Pr}\left(u_{i}=0\right)=$ $\operatorname{Pr}\left(y_{i}<\epsilon_{i} \mid \mathcal{H}_{0}\right) \operatorname{Pr}\left(\mathcal{H}_{0}\right)+\operatorname{Pr}\left(y_{i}<\epsilon_{i} \mid \mathcal{H}_{1}\right) \operatorname{Pr}\left(\mathcal{H}_{1}\right)=$ $\left(1-p_{f_{i}}\right) \operatorname{Pr}\left(\mathcal{H}_{0}\right)+\left(1-p_{d_{i}}\right) \operatorname{Pr}\left(\mathcal{H}_{1}\right)=1-p_{f_{i}} \operatorname{Pr}\left(\mathcal{H}_{0}\right)-$ $p_{d_{i}} \operatorname{Pr}\left(\mathcal{H}_{1}\right)$. Similarly, $\operatorname{Pr}\left(u_{i}=1\right)=p_{f_{i}} \operatorname{Pr}\left(\mathcal{H}_{0}\right)+$ $p_{d_{i}} \operatorname{Pr}\left(\mathcal{H}_{1}\right)$. It is noted that for the case of $\operatorname{Pr}\left(\mathcal{H}_{0}\right)=$ $\operatorname{Pr}\left(\mathcal{H}_{1}\right)$ and having $p_{f_{i}}+p_{d_{i}} \cong 1$ when the signal-to-noise ratios of the sensing links are low [3], the threshold in (26) is approximately 1 . Note also that each density function in the above LRT is the density of a zero-mean complex Gaussian variable. As such, the LRT is determined by $\mathcal{E}\left(\left|\hat{v}_{\boldsymbol{h}, i}\right|^{2} \mid u_{\boldsymbol{i}}=1\right)$ and $\mathcal{E}\left(\left|\hat{v}_{\boldsymbol{h}, \boldsymbol{i}}\right|^{2} \mid u_{\boldsymbol{i}}=0\right)$. These two expectations are computed as follows. First, according to (23), one has

$$
\mathcal{E}\left(\left|\hat{v}_{\boldsymbol{h}, i}\right|^{2} \mid u_{i}=1\right)=\left[\boldsymbol{C} \mathcal{K}_{i, 1} \boldsymbol{C}\right]_{i, i} \triangleq \zeta_{i, 1},
$$

where

$$
\mathcal{K}_{i, 1}=\mathcal{E}\left(z z^{H} \mid u_{i}=1\right)=\boldsymbol{G} \overline{\boldsymbol{D}}_{i, 1} \boldsymbol{G}^{T}+\sigma_{\mathrm{FC}}^{2} \boldsymbol{I}
$$

and $\left[\overline{\boldsymbol{D}}_{i, 1}\right]_{k, k}=\bar{d}_{k}, k \neq i$, and $\left[\overline{\boldsymbol{D}}_{i, 1}\right]_{i, i}=\frac{\overline{\boldsymbol{d}}_{i}}{\kappa_{i}}$. Similarly,

$$
\mathcal{E}\left(\left|\hat{v}_{\boldsymbol{h}, i}\right|^{2} \mid u_{i}=0\right)=\left[\boldsymbol{C} \mathcal{K}_{i, 0} \boldsymbol{C}\right]_{i, i} \triangleq \zeta_{i, 0}
$$

with

$$
\mathcal{K}_{i, 0}=\mathcal{E}\left(z z^{H} \mid u_{i}=0\right)=\boldsymbol{G} \overline{\boldsymbol{D}}_{i, 0} \boldsymbol{G}^{T}+\sigma_{\mathrm{FC}}^{2} \boldsymbol{I}
$$


and $\left[\overline{\boldsymbol{D}}_{i, 0}\right]_{k, k}=\bar{d}_{k}, k \neq i$, and $\left[\overline{\boldsymbol{D}}_{i, 0}\right]_{i, i}=0$. Simplifying (26) gives

$$
\left|\hat{v}_{\boldsymbol{h}, i}\right|^{2} \underset{\hat{u}_{i}=0}{\hat{u}_{i=1}} \frac{\ln \left(\frac{1-p_{f_{i}} \operatorname{Pr}\left(\mathcal{H}_{0}\right)-p_{d_{i}} \operatorname{Pr}\left(\mathcal{H}_{1}\right)}{p_{f_{i}} \operatorname{Pr}\left(\mathcal{H}_{0}\right)+p_{d_{i}} \operatorname{Pr}\left(\mathcal{H}_{1}\right)} \cdot \frac{\zeta_{i, 1}}{\zeta_{i, 0}}\right)}{\frac{\zeta_{i, 1}-\zeta_{i, 0}}{\zeta_{i, 1} \zeta_{i, 0}}} \triangleq \varrho_{i} .
$$

The reliability of the hard decision rule in (31) concerning the bit sent by the $i$ th $C R$ can be evaluated by the following set of correct/error probabilities:

$$
\begin{gathered}
\varpi_{i}=\operatorname{Pr}\left(\hat{u}_{i}=1 \mid u_{i}=1\right)=\operatorname{Pr}\left(\left|\hat{v}_{\boldsymbol{h}, i}\right|^{2}>\varrho_{i} \mid u_{i}=1\right)=\mathrm{e}^{-\frac{\varrho_{i}}{\zeta_{i, 1}}}, \\
\vartheta_{i}=\operatorname{Pr}\left(\hat{u}_{i}=1 \mid u_{i}=0\right)=\operatorname{Pr}\left(\left|\hat{v}_{\boldsymbol{h}, i}\right|^{2}>\varrho_{i} \mid u_{i}=0\right)=\mathrm{e}^{-\frac{\varrho_{i}}{\zeta_{i, 0}}} .
\end{gathered}
$$

Note that $\operatorname{Pr}\left(\hat{u}_{i}=0 \mid u_{i}=1\right)=1-\varpi_{i}$ and $\operatorname{Pr}\left(\hat{u}_{i}=0 \mid u_{i}=0\right)=1-\vartheta_{i}$.

The next processing step in the fusion center is to combine the hard decisions $\hat{u}_{i}, i=1, \ldots, K$, to make a final sensing decision. This can be done in the same manner as in [3] by forming the following likelihood ratio for the decoded bits:

$$
\frac{\operatorname{Pr}\left(\hat{\boldsymbol{u}} \mid \mathcal{H}_{1}\right)}{\operatorname{Pr}\left(\hat{\boldsymbol{u}} \mid \mathcal{H}_{0}\right)}=\frac{\prod_{i=1}^{K}\left(1-\wp_{d_{i}}\right)^{\left(1-\hat{u}_{i}\right)} \wp_{d_{i}}^{\hat{u}_{i}}}{\prod_{i=1}^{K}\left(1-\wp_{i}\right)^{\left(1-\hat{u}_{i}\right)} \wp_{f_{i}}^{\hat{u}_{i}}}
$$

where $\wp_{d i}=\operatorname{Pr}\left(\hat{u}_{i}=1 \mid \mathcal{H}_{1}\right)$ and $\wp_{i}=\operatorname{Pr}\left(\hat{u}_{i}=1 \mid \mathcal{H}_{0}\right)$ are the probabilities of detection and probabilities of false alarm associated with the decoded bits $\hat{u}_{i}$. They are shown in [3] to be given as $\wp_{d_{i}}=p_{d_{i}} \varpi_{i}+\left(1-p_{d_{i}}\right) \vartheta_{i}$ and $\wp_{f_{i}}=$ $p_{f_{i}} \varpi_{i}+\left(1-p_{f_{i}}\right) \vartheta_{i}$.

Working with the logarithm of (34), the decoding-based fusion rule is given as [3]

$$
L^{(\mathrm{D})}(\hat{\boldsymbol{u}})=\sum_{i=1}^{K}\left[\varphi_{0, i}\left(1-\hat{u}_{i}\right)+\varphi_{1, i} \hat{u}_{i}\right] \underset{\mathcal{H}_{0}}{\stackrel{\mathcal{H}_{1}}{\gtrless}} \lambda^{(\mathrm{D})},
$$

where $\lambda^{(D)}$ is the threshold, while the weights are $\varphi_{0, i}=$ $\log \frac{1-\wp_{d i}}{1-\wp_{f_{i}}}$ and $\varphi_{1, i}=\log \frac{\wp_{d i}}{\wp_{i}}$. Note that the above fusion rule is simply a weighted linear combination of the hard-decision bits. Moreover, the weights are inherently adjusted according to both the decision of the $i$ th CR and the quality of the reporting channel.

By approximating $L^{(\mathrm{D})}(\hat{\boldsymbol{u}})$ as a Gaussian random variable under each hypothesis, the expressions of the probability of false alarm and the probability of detection at the fusion center are as follows:

$$
P_{\mathrm{F}}^{(\mathrm{D})}\left(\boldsymbol{p}_{\boldsymbol{f}}, \lambda^{(\mathrm{D})}\right)=\operatorname{Pr}\left(L^{(\mathrm{D})}(\hat{\boldsymbol{u}})>\lambda^{(\mathrm{D})} \mid \mathcal{H}_{0}\right)=Q\left(\frac{\lambda^{(\mathrm{D})}-m_{0}}{\delta_{0}}\right)
$$

and

$$
P_{\mathrm{D}}^{(\mathrm{D})}\left(\boldsymbol{p}_{\boldsymbol{d}}, \lambda^{(\mathrm{D})}\right)=\operatorname{Pr}\left(L^{(\mathrm{D})}(\hat{\boldsymbol{u}})>\lambda^{(\mathrm{D})} \mid \mathcal{H}_{1}\right)=Q\left(\frac{\lambda^{(\mathrm{D})}-m_{1}}{\delta_{1}}\right) \text {. }
$$

The mean values, $m_{0}$ and $m_{1}$, and the variances, $\delta_{0}^{2}$ and $\delta_{1}^{2}$, can be determined in a similar fashion as in [3]. The means are given as

$$
\begin{aligned}
& m_{0}=\mathcal{E}\left[L^{(\mathrm{D})}(\hat{\boldsymbol{u}}) \mid \mathcal{H}_{0}\right]=\sum_{i=1}^{K} m_{i, 0} \\
& m_{i, 0}=\left[\varpi_{i} p_{f_{i}}+\vartheta_{i}\left(1-p_{f_{i}}\right)\right]\left[\varphi_{1, i}-\varphi_{0, i}\right]+\varphi_{0, i}
\end{aligned}
$$

and

$$
\begin{aligned}
& m_{1}=\mathcal{E}\left[L^{(\mathrm{D})}(\hat{\boldsymbol{u}}) \mid \mathcal{H}_{1}\right]=\sum_{i=1}^{K} m_{i, 1} \\
& m_{i, 1}=\left[\varpi_{i} p_{d_{i}}+\vartheta_{i}\left(1-p_{d_{i}}\right)\right]\left[\varphi_{1, i}-\varphi_{0, i}\right]+\varphi_{0, i} .
\end{aligned}
$$

Using the fact that $\mathcal{E}\left[\hat{u}_{i} \mid \mathcal{H}_{0}\right]=\wp_{f_{i}}$ and $\mathcal{E}\left[\hat{u}_{i} \mid \mathcal{H}_{1}\right]=\wp_{d_{i}}$, the variances are

$$
\begin{aligned}
\delta_{0}^{2}= & \operatorname{Var}\left[L^{(\mathrm{D})}(\hat{\boldsymbol{u}}) \mid \mathcal{H}_{0}\right]=\sum_{i=1}^{K} \mathcal{E}\left[\hat{u}_{i}^{2} \mid \mathcal{H}_{0}\right]\left[\varphi_{1, i}-\varphi_{0, i}\right]^{2} \\
& +\sum_{i, j=1, i \neq j}^{K}\left[\varphi_{1, i}-\varphi_{0, i}\right]\left[\varphi_{1, j}-\varphi_{0, j}\right] \mathcal{E}\left[\hat{u}_{i} \hat{u}_{j} \mid \mathcal{H}_{0}\right]-m_{0}^{2} \\
= & \sum_{i=1}^{K} \wp_{f_{i}}\left[\varphi_{1, i}-\varphi_{0, i}\right]^{2}+\sum_{i, j=1, i \neq j}^{K}\left[\varphi_{1, i}-\varphi_{0, i}\right]\left[\varphi_{1, j}\right. \\
& \left.-\varphi_{0, j}\right] \mathcal{C}_{0}(i, j)-m_{0}^{2}
\end{aligned}
$$

and

$$
\begin{aligned}
\delta_{1}^{2}= & \operatorname{Var}\left[L^{(\mathrm{D})}(\hat{\boldsymbol{u}}) \mid \mathcal{H}_{1}\right]=\sum_{i=1}^{K} \mathcal{E}\left[\hat{u}_{i}^{2} \mid \mathcal{H}_{1}\right]\left[\varphi_{1, i}-\varphi_{0, i}\right]^{2} \\
& +\sum_{i, j=1, i \neq j}^{K}\left[\varphi_{1, i}-\varphi_{0, i}\right]\left[\varphi_{1, j}-\varphi_{0, j}\right] \mathcal{E}\left[\hat{u}_{i} \hat{u}_{j} \mid \mathcal{H}_{1}\right]-m_{1}^{2} \\
= & \sum_{i=1}^{K} \wp_{d_{i}}\left[\varphi_{1, i}-\varphi_{0, i}\right]^{2}+\sum_{i, j=1, i \neq j}^{K}\left[\varphi_{1, i}-\varphi_{0, i}\right]\left[\varphi_{1, j}\right. \\
& \left.-\varphi_{0, j}\right] \mathcal{C}_{1}(i, j)-m_{1}^{2} .
\end{aligned}
$$

Observe that, different from the case of orthogonal transmission considered in [3], the above variances depend on the correlations of the decoded bits, $\hat{u}_{i}$ and $\hat{u}_{j}$, under the two hypotheses. These correlations are determined as follows:

$$
\begin{aligned}
\mathcal{C}_{0}(i, j) \triangleq \mathcal{E}\left[\hat{u}_{i} \hat{u}_{j} \mid \mathcal{H}_{0}\right] & =\operatorname{Pr}\left(\left|\hat{v}_{\boldsymbol{h}, i}\right|^{2}>\varrho_{i},\left|\hat{v}_{\boldsymbol{h}, j}\right|^{2}>\varrho_{j} \mid \mathcal{H}_{0}\right) \\
& =\mathrm{e}^{-\varrho_{i j}^{\mathrm{T}}\left(\left[\zeta_{0}(i, j)\right]\right)^{-1} \varrho_{i j}}
\end{aligned}
$$




$$
\begin{aligned}
\mathcal{C}_{1}(i, j) \triangleq \mathcal{E}\left[\hat{u}_{i} \hat{u}_{j} \mid \mathcal{H}_{1}\right] & =\operatorname{Pr}\left(\left|\hat{v}_{\boldsymbol{h}, i}\right|^{2}>\varrho_{i},\left|\hat{v}_{\boldsymbol{h}, j}\right|^{2}>\varrho_{j} \mid \mathcal{H}_{1}\right) \\
& =\mathrm{e}^{-\varrho_{i j}^{\mathrm{T}}\left[\left(\zeta_{1}(i, j)\right]\right)^{-1} \varrho_{i j}},
\end{aligned}
$$

where $\varrho_{i j} \triangleq\left[\varrho_{i}, \varrho_{j}\right]^{T}, \zeta_{0} \triangleq \boldsymbol{G D}_{0} \boldsymbol{G}^{T}+\sigma_{\mathrm{FC}}^{2} \boldsymbol{I}$, and $\zeta_{1} \triangleq$ $\boldsymbol{G} \boldsymbol{D}_{1} \boldsymbol{G}^{T}+\sigma_{\mathrm{FC}}^{2} \boldsymbol{I}$.

Recall that an approximation of the optimal sensing thresholds used at the cognitive radios for the energybased fusion rule is given in (21). The same result applies to the decoding-based fusion rule presented in this section. The proof of this follows the same steps in Appendix C of [3].

The next section compares the performance of the energy-based and decoding-based fusion rules and also verifies the accuracy of our analysis. It is pointed out that the simple expressions of the probability of false alarm and the probability of detection (Equations (18) and (19) for the energy-based fusion rule, or (36) and (37) for the decoding-based fusion rule) are very convenient not only in determining the threshold at the fusion center $\left(\lambda^{(E)}\right.$ or $\left.\lambda^{(D)}\right)$ for a given target probability of false alarm (or probability of detection), but also in evaluating the performance of different sets of signature vectors used in nonorthogonal transmission of local decisions.

\section{Simulation results}

Each point in the simulations results is obtained by averaging over $10^{4}$ random realizations for primary transmitted signal, fading channels, and noise. For the sake of simplicity, it is assumed that $\operatorname{Pr}\left(\mathcal{H}_{0}\right)=\operatorname{Pr}\left(\mathcal{H}_{1}\right)=0.5$. Unless otherwise stated, the number of the CRs is set to $K=30$, the number of samples taken is $N=500$, and the set of WBE signature sequences is assigned to CR users.

Figures 2 and 3 depict the probabilities of detection versus the probabilities of false alarm when the energy-based and decoding-based fusion rules are used, respectively. For both figures, all the sensing channels have the same average SNR of $\gamma_{i}=\gamma=-15 \mathrm{~dB}$, and also, all the reporting channels have the same SNR of $\xi_{i}=\xi=$ $20 \mathrm{~dB}$. The sensing threshold is chosen as $\epsilon_{i}=\epsilon^{*}=$ $\left(1+\frac{1}{\gamma}\right) \log (\gamma+1)$ at each CR node. For the energybased fusion rule, it is clearly seen from Figure 2 that by increasing the length of signature vectors from $M=$ 5 to $M=15$, the probability of detection versus the probability of false alarm increases significantly, whereas increasing $M$ beyond 15 only improves the sensing performance slightly. This suggests that compared to the case of orthogonal transmission, the transmission bandwidth for the reporting phase can be reduced by half by using WBE sequences with $M=15$ while not loosing too much the sensing performance. It can also be observed that the analytical and simulation results match very well ${ }^{\mathrm{a}}$. For

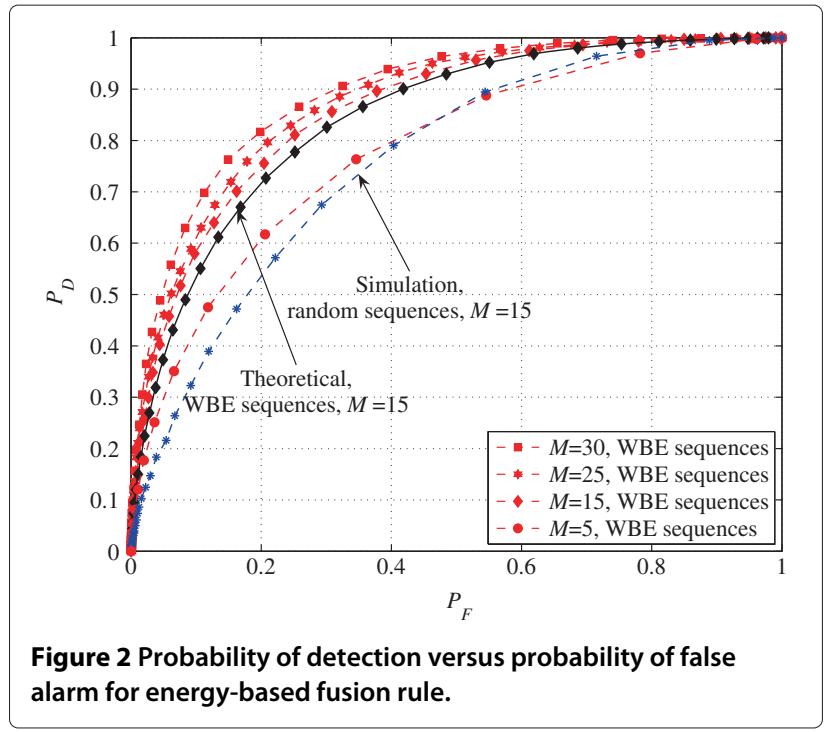

comparison purpose, the performance with random signature vectors was also obtained and plotted in Figure 2 for $M=15$. Specifically, the result is obtained by averaging over $10^{4}$ random realizations of the signature vectors. As expected, the performance of using WBE sequences significantly outperforms the performance with random sequences.

On the other hand, for the proposed decoding-based fusion rule, Figure 3 shows that the sensing performance is significantly improved not only when increasing $M$ from 5 to 15 , but also by further increasing $M$ to 30 . Such an improvement can be explained by the fact that detection of the transmitted bits from CRs strongly depends on the interference caused by nonorthogonal transmission. As the interference reduces with longer signature vectors

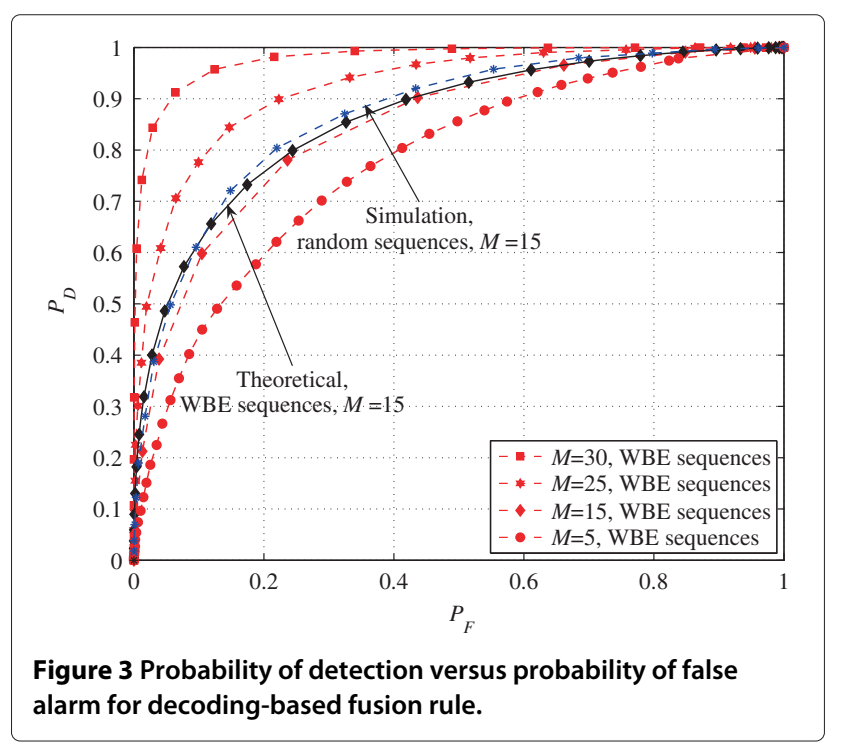


(larger $M$ ), the detection performance is greatly enhanced when all the reporting channels are fairly strong. The figure also shows that the theoretical result (with $M=15$ ) follows the simulation result closely. Another observation from Figure 3 is that with random signature vectors, the sensing performance is slightly better than the performance with WBE sequences. As pointed out before, it is difficult to obtain the optimal set of signature vectors for the proposed decoding-based fusion rule. The results in Figure 3 suggest that either randomly generated or WBE sequences can be used for the proposed decoding-based fusion rule.

Figure 4 compares the probabilities of detection obtained by simulation with the energy-based and decoding-based fusion rules for different values of $M$ and when the WBE sequences are used. As can be seen, even in the presence of strong reporting channels, the energybased fusion rule performs equally or better than the decoding-based fusion rule when $M \leq 15$. On the contrary, for $M>15$, the decoding-based fusion rule outperforms the energy-based fusion rule. The obtained results suggest that the energy-based fusion rule can be employed as an efficient fusion rule in cognitive radio applications under very limited transmission bandwidth (i.e., $M \leq 15$ ).

Figures 5 and 6 present the performance comparison between the two sensing algorithms for the situations when the SNRs are different at the CRs and/or the FC. In the first scenario, all the CRs experience the same 'sensing' SNR of $\gamma=\gamma_{i}=-15 \mathrm{~dB}$ from the primary user, but each group of CRs transmits its sensing results to the fusion center with the 'reporting' SNRs of $-5 \mathrm{~dB}$ (6 CRs), $-1 \mathrm{~dB}$ (6 CRs), $0 \mathrm{~dB}$ (12 CRs), and $5 \mathrm{~dB}$ (6 CRs). In the second scenario, the CRs are divided into six groups of five CRs each.The sensing SNRs are given

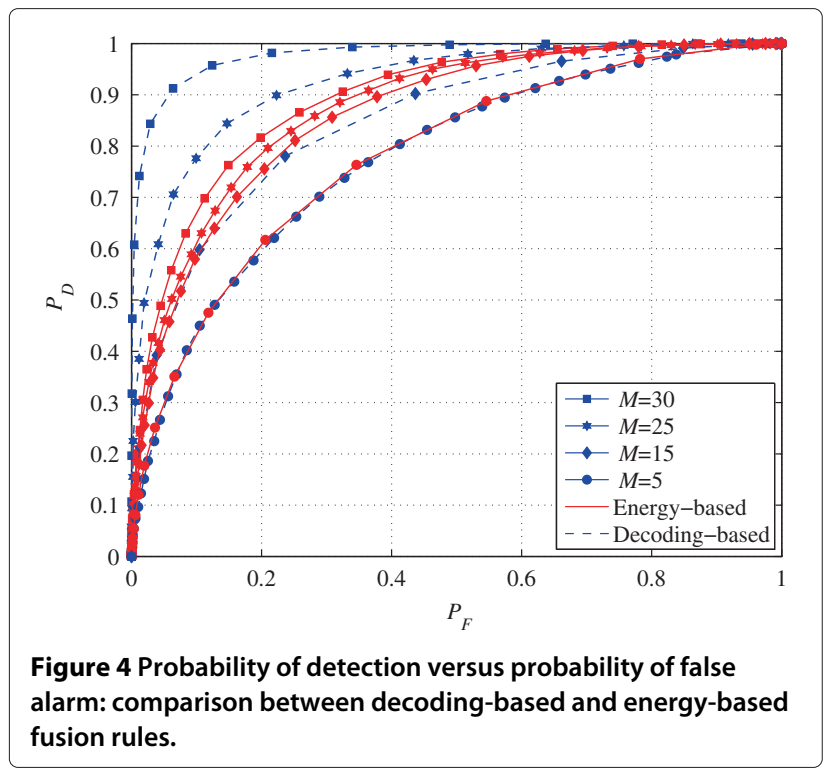

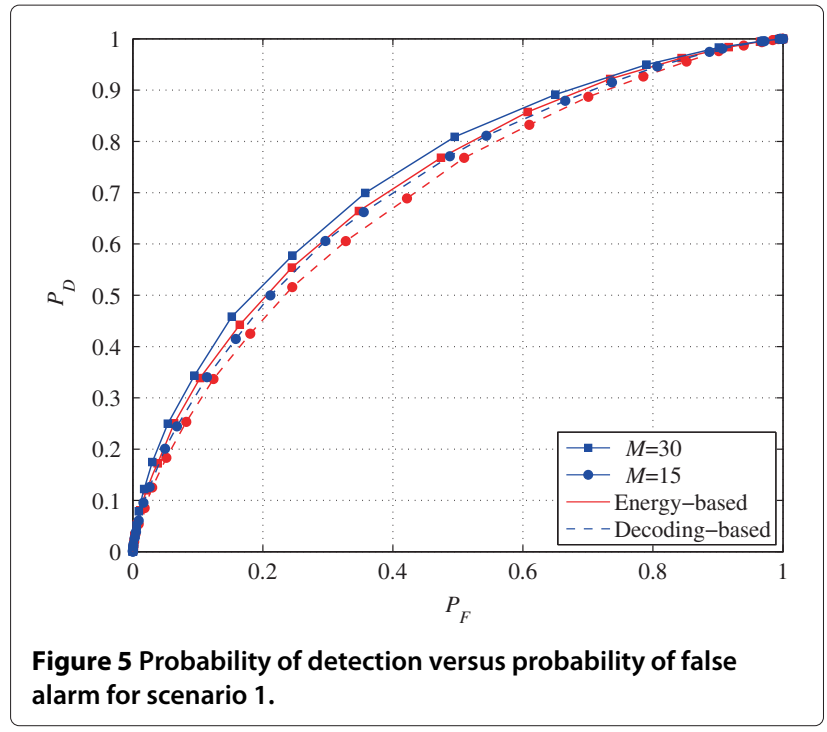

as $\{-15,-24,-5,-10,-20\} \mathrm{dB}$, whereas the reporting SNRs are considered to be $\{5,10,1,1,5\}$ dB.In comparison to the first scenario, the second scenario has stronger reporting channels.

As can be seen in Figures 5 and 6, both figures show performance improvement when $M$ increases from 15 to 30. However, for the first scenario with the weak reporting links, the improvement is marginal. This is because the sensing performance in this scenario is mostly affected by the noise at the FC rather than the interference caused by shorter signature vectors. Another important observation is that the energy-based fusion rule outperforms the decoding-based rule. Thus, it can be concluded that for low channel SNRs, the energy-based fusion rule is preferred over the decoding-based fusion rule due to its lower

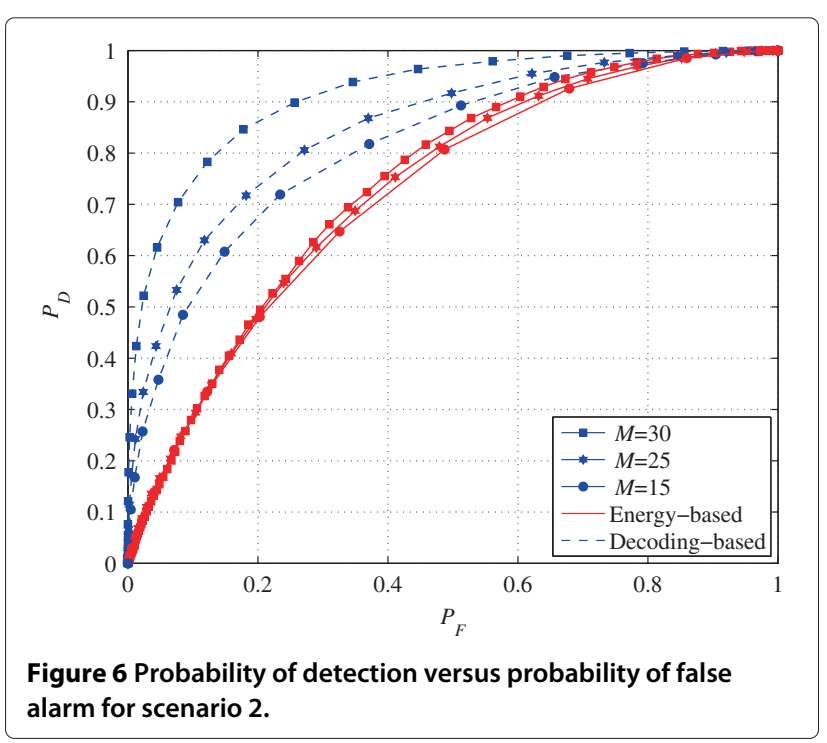


complexity while at the same time delivering equal or better performance.

For the second scenario, when the channels are fairly strong, the decoding-based detection significantly outperforms the energy-based detection. Also, as $M$ increases, the performance of the decoding-based fusion rule quickly improves due to the reduction of interference. However, for the energy-based fusion rule, interference reduction does not play a significant role in improving the sensing performance.

Figure 7 presents a comparison between the probabilities of detection of the two sensing algorithms for the situation when $N=500$ and $P_{\mathrm{F}}$ is fixed at 0.05 at the fusion center. The sensing SNRs are -12 and $-9 \mathrm{~dB}$ at the CRs, and the reporting SNRs are $20 \mathrm{~dB}$. As can be seen, for a lower sensing SNR, the performance of both algorithms improves significantly with increasing $M$. Such an improvement can be explained by the fact that as $M$ increases, the interference caused by nonorthogonal transmission is reduced, which helps to enhance the sensing quality. On the other hand, when the sensing SNR increases, the individual CR sensing performance quickly improves, which makes the overall desirable sensing performance achievable by using shorter-length signature vectors. For example, given the target $P_{\mathrm{F}}=0.05$ and the sensing time of 500 samples, in order to achieve a target $P_{\mathrm{D}}=0.9$ when the sensing SNRs are $-12 \mathrm{~dB}$, it is seen that setting $M=15$ is sufficient. The use of such shorter signature vectors translates to half of the bandwidth as required by orthogonal signature vectors.

Finally, in Figure 8, the normalized throughput, defined as $\left(1-\frac{N}{T}\right)\left(1-P_{\mathrm{F}}\right)$, is plotted versus the entire time frame of $1 \mathrm{~ms}$ (which is equivalent to $T=1,000$ samples). Here, the threshold $\lambda^{(D)}$ is selected to fix the probability of detection at $P_{\mathrm{D}}=0.8$. Two sets of signature vectors, whose lengths are $M=25$ and $M=10$, are considered.

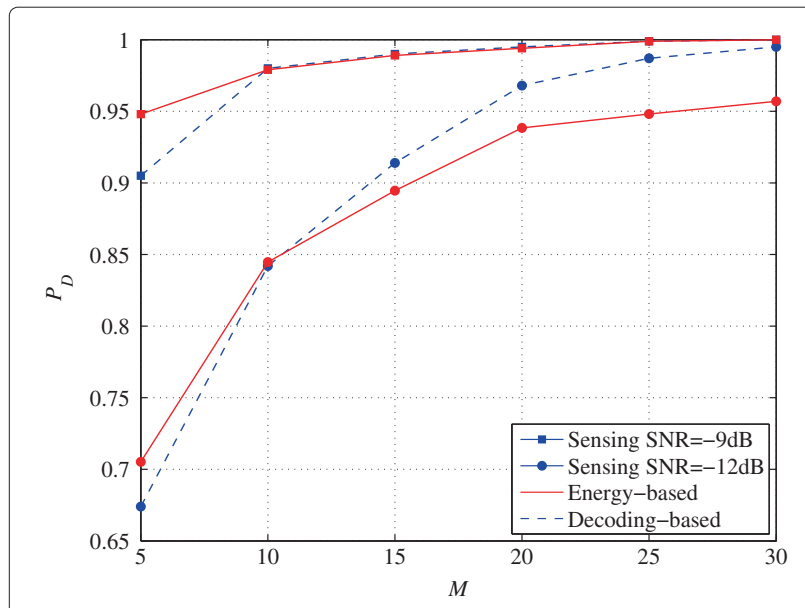

Figure 7 Probability of detection versus $M$ for $\boldsymbol{P}_{\mathrm{F}}=\mathbf{0 . 0 5}$.

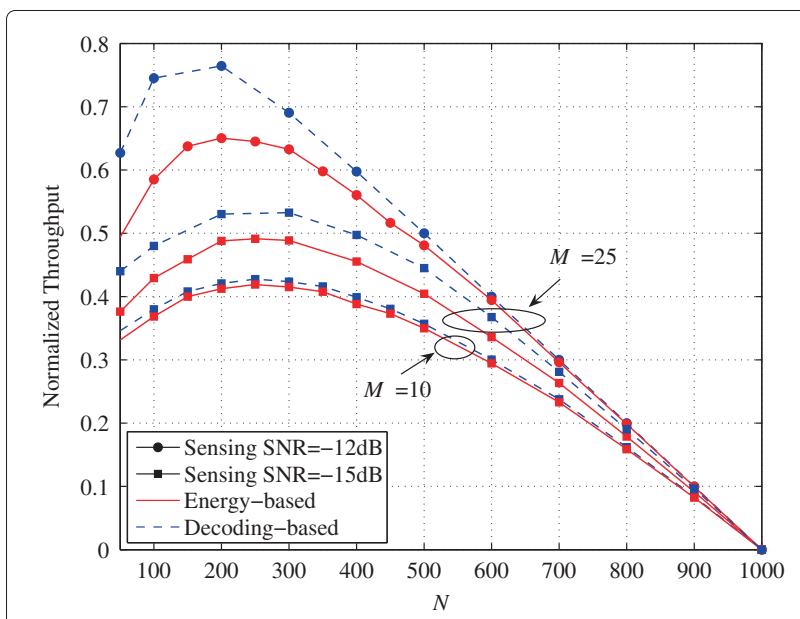

Figure 8 Normalized throughput versus time samples $N$ for $P_{\mathrm{D}}=0.8$.

For the given target $P_{\mathrm{D}}$ and for the case $M=25$, it can be seen that the decoding-based fusion rule achieves a higher secondary throughput than the energy-based fusion rule. However, for the case $M=10$, both the decodingbased and energy-based fusion rules achieve almost the same secondary throughput. These results are in agreement with our previous discussion concerning Figure 4. Another important observation of Figure 8 is that the throughput functions are concave and attain a maximum point at a certain sensing time. This means that for a given target $P_{\mathrm{D}}$, an optimal sensing time can be found to maximize the secondary throughput. Alternatively, for a given target $P_{\mathrm{D}}$, the minimum length of the signature vectors can be determined to achieve a target secondary throughput within the allowable sensing time.

\section{Conclusions}

This paper has proposed a low-complexity decodingbased fusion rule for cognitive radio networks with nonorthogonal transmission of local decisions in the presence of channel impairments and noise. The proposed fusion rule first performs the MMSE estimation of the transmitted information, makes decisions on the individual bits sent by the cognitive radios, and then combines these hard-decision bits in a linearly weighted manner. Performance comparison with the energy-based fusion rule shows the superiority of the proposed fusion rule when the reporting channels are reasonably strong. The excellent match between simulation and analytical results verify the accuracy of the performance analysis of the proposed fusion rule.

\section{Endnote}

a To avoid having too many curves on the same figure, only the analytical result for the case of $M=15$ is shown. 


\section{Competing interests}

The authors declare that they have no competing interests.

\section{Acknowledgements}

This work was supported by an NSERC Discovery Grant.

\section{Author details}

1 Department of Electrical and Computer Engineering, University of Manitoba, 75A Chancellor's Circle, Winnipeg, Manitoba R3T 5V6, Canada. ${ }^{2}$ Department of Electrical and Computer Engineering, University of Saskatchewan, 57 Campus Drive, Saskatoon, Saskatchewan S7N 5A9, Canada.

Received: 4 April 2013 Accepted: 11 June 2013

Published: 8 July 2013

\section{References}

1. S Yiu, R Schober, Nonorthogonal transmission and noncoherent fusion of censored decisions. IEEE Trans. Veh. Technol. 58, 263-273 (2009)

2. HH Nguyen, E Shwedyk, Bandwidth-constrained signature waveforms and Walsh signal space receivers for synchronous CDMA systems. IEEE Trans. Commun. 50(7), 1137-1149 (2002)

3. S Bokharaiee, HH Nguyen, E Shwedyk, Cooperative spectrum sensing in cognitive radio networks with noncoherent transmission. IEEE Trans Veh. Technol. 61(3), 2476-2489 (2012)

4. H Urkwitz, Energy detection of unknown deterministic signals. Proc. IEEE. 55, 523-531 (1967)

5. C Shannon, Communication in the presence of noise. Proc. IEEE. 72(9), 1192-1201 (1984)

6. HH Nguyen, EShwedyk, A First Course in Digital Communications (Cambridge University Press, Cambridge, 2009)

7. Z Quan, S Cui, AH Sayed, HV Poor, Optimal multiband joint detection for spectrum sensing in cognitive radio networks. IEEE Trans. Signal Process. 57, 1128-1140 (2009)

8. YC Liang, Y Zeng, EC Peh, AT Hoang, Sensing-throughput tradeoff for cognitive radio networks. IEEE Trans. Wireless Commun. 7(4), 1326-1337 (2008)

9. Z Quan, S Cui, AH Sayed, Optimal linear cooperation for spectrum sensing in cognitive radio networks. IEEE J. Select. Topics Signal Process. 2(1), 28-40 (2008)

10. S Kim, J Lee, H Wang, D Hong, Sensing performance of energy detector with correlated multiple antennas. IEEE Signal Process. Lett. 16, 671-674 (2009)

11. L Welch, Lower bounds on the maximum cross correlation of signals. IEEE Trans. Inform. Theory. 20(3), 397-399 (1974)

12. P Viswanath, V Anantharam, D Tse, Optimal sequences, power control, and user capacity of synchronous CDMA systems with linear MMSE multiuser receivers. IEEE Trans. Inform. Theory. 45(6), 1968-1983 (1999)

\section{doi:10.1186/1687-1499-2013-184}

Cite this article as: Bokharaiee et al.: A decoding-based fusion rule for cooperative spectrum sensing with nonorthogonal transmission of local decisions. EURASIP Journal on Wireless Communications and Networking 2013 2013:184.

\section{Submit your manuscript to a SpringerOpen ${ }^{\mathcal{O}}$ journal and benefit from:}

- Convenient online submission

- Rigorous peer review

- Immediate publication on acceptance

- Open access: articles freely available online

- High visibility within the field

- Retaining the copyright to your article

Submit your next manuscript at $\boldsymbol{\wedge}$ springeropen.com 